\title{
Situación epidemiológica de la infección por VIH y el síndrome de inmunodeficiencia adquirida en Chile
}

\author{
Paulina Martínez G., Andrea Olea N. y Mónica Chiu A.
}

\section{Epidemiology of HIV infection and acquired immunedeficiency disease syndrome in Chile}

Since 1984 to December 2004, 14.611 HIV/AIDS cases (85\% in males) have been reported to the Chilean surveillance system. This figure represents an incidence of $103 \times 10^{5}$ inhabitants. The epidemic affects mainly men in their active working and sexual age. The main risk factor for infection, has been the sexual contact. Universal access to antiretroviral therapy (ART) has changed the epidemic trend. In 1997 bi-therapy and in 2003 three-drug therapy (HAART) were implemented. Mortality was 2,4 cases x $10^{5}$ inhabitants during 2004 , with a $67 \%$ reduction since 1984 . Vertical transmission has been significantly reduced, as a result of implementing since 1995 a preventive strategy that benefits seropositive women and their newborn infant. Local experts are preparing an electronic registration system in order to optimize resources and to accede to statistics in a real time.

Key words: Immunodeficiency virus, AIDS, epidemiology, Chile.

Palabras claves: Virus de inmunodeficiencia humana, SIDA, epidemiología, Chile.

\section{Situación epidemiológica mundial}

$\mathrm{S}$ e estima que en el mundo viven actualmente sobre 40 millones de personas infectadas por VIH/SIDA; sólo en el año 2005 contrajeron el virus 5 millones de personas (ONUSIDA/OMS, 2004).

África subsahariana sigue siendo la región más afectada, con aproximadamente 25,8 millones de personas infectadas con VIH -las dos terceras partes del total de personas afectadas por el virus en el mundo- $\mathrm{y}$ $77 \%$ del total de mujeres con infección por VIH. En esta región fallecieron aproximadamente 2,4 millones de personas en el año 2005, mientras que otros 3,2 millones contrajeron la infección (ONUSIDA/OMS, 2004).

Hay epidemias en rápido crecimiento en Europa oriental, Asia central, y en Asia oriental. En las primeras, el número de personas afectadas por el VIH ha aumentado hasta 1,6 millones desde 2003, y el número de fallecidos por SIDA casi se ha duplicado en el mismo período. En Asia oriental, el número de personas con infección por VIH en el 2005 aumentó en un quinto en comparación con los dos años anteriores (ONUSIDA/OMS, 2004).
En América Latina y el Caribe se estima actualmente que más de 2 millones de personas padecen de la infección por VIH/SIDA. En 12 países de la región la prevalencia es a lo menos de $1 \%$. La principal modalidad de transmisión en Latinoamérica sigue siendo por relaciones sexuales entre hombres, seguida por las prácticas asociadas al uso de drogas inyectables, apreciándose desde los años 90 un incremento de la transmisión heterosexual. Sin embargo, existe gran variabilidad del patrón de transmisión al interior de la región. Un ejemplo de esto es el caso de Argentina, donde la categoría de exposición mayoritaria es a través de compartir jeringas durante la práctica de uso de drogas inyectables. Para varios países de la zona constituye una preocupación la relación entre drogas y VIH, en el entendido que el consumo de drogas por cualquier vía favorece conductas de riesgo (ONUSIDA/OMS, 2004) (Figura 1).

\section{Situación epidemiológica nacional}

En Chile, la vigilancia epidemiológica de infección por VIH y SIDA está regulada por el Decreto Supremo $\mathrm{N}^{\circ} 158$ y se realiza mediante vigilancia pasiva, es decir, mediante recolección de datos de casos notificados
Ministerio de Salud de Chile Departamento de Epidemiología División de Planificación Sanitaria Subsecretaría de Salud Pública

Recibido: 30 junio 2006 Aceptado: 25 julio 2006

Correspondencia a: Paulina Martínez Gallardo pmartinez@minsal.gov.cl 
Tabla. Resumen mundial de la epidemia de infección por VIH y de SIDA, Diciembre de 2005

Personas infectadas por VIH en 2005

Total

Adultos

Mujeres

Bajo 15 años de edad

40,3 millones (36,7 - 45,3 millones) 38,0 millones $(34,5-42,6$ millones)

17,5 millones $(16,2-19,3$ millones $)$ 2,3 millones $(2,1-2,8$ millones $)$

Nuevas infecciones por VIH en 2005

Total

Adultos

Bajo 15 años de edad

4,9 millones $(4,3-6,6$ millones $)$

4,2 millones (3,6 - 5,8 millones)

$700000(630.000-820.000)$

Defunciones causadas por el SIDA en 2005

Total

Adultos

Bajo 15 años de edad

3,1 millones (2,8 - 3,6 millones)

2,6 millones $(2,3-2,9$ millones $)$

$570000(510.000-670.000)$

Los márgenes de variación de las estimaciones presentadas en este cuadro definen los límites dentro de los que se encuentran las cifras reales, y se basan en la mejor información disponible. 00003-Sp-1-Diciembre 2005

Fuente: ONUSIDA/Organización Mundial de la Salud.

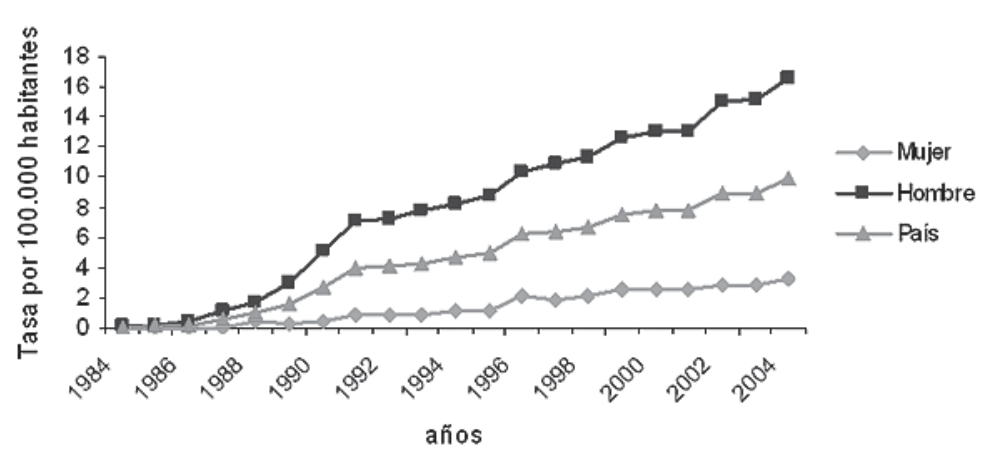

Figura 1. Tasa de casos notificados de infección por VIH/SIDA por sexo. Chile, 1984-2004.

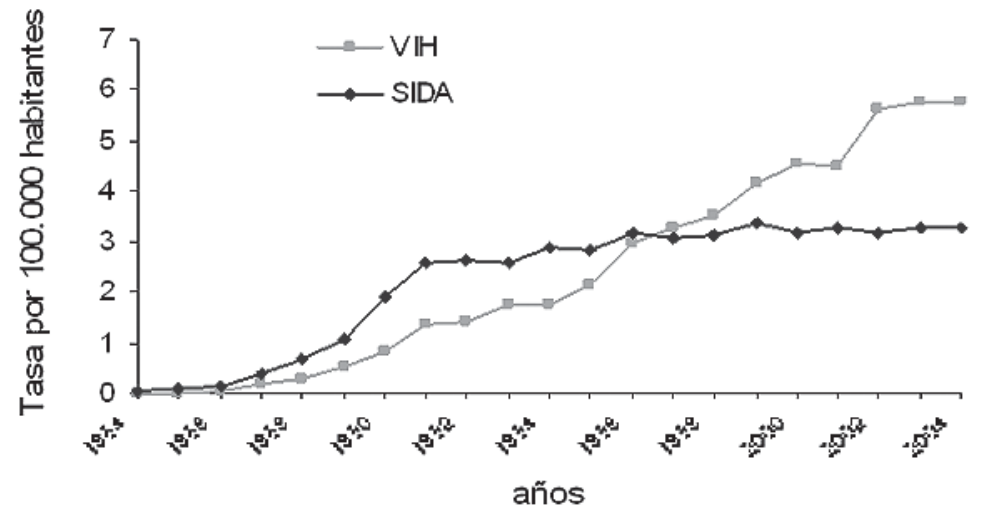

Figura 2. Tasa de notificación de casos de infección por VIH y SIDA. Chile, 1984-2004. por el Boletín ENO (Enfermedades de Notificación Obligatoria), "Encuesta epidemiológica de caso de infección por VIH/SIDA" y certificados de defunción.

\section{Metodología}

Se analizó la base de datos de infección por VIH/ SIDA del Ministerio de Salud entre 1984 y 2004, que contiene toda la información proveniente de la "Encuesta epidemiológica de caso de infección por VIH/ SIDA". Se calcularon las tasas de incidencia y defunción por 100.000 habitantes.

También fueron consideradas otras fuentes de información: bases de datos de muertes (DEIS) y estadísticas de población del Instituto nacional de Estadísticas (INE).

\section{Magnitud y tendencia}

A la fecha de elaboración de este artículo, entre 1984 y diciembre de 2004 se registraron 14.611 casos, de las trece regiones del país. En la actualidad 6.840 tienen SIDA y 7.558 son personas asintomáticas. La tasa de incidencia de casos notificados VIH/SIDA para el período 1984-2004, es de 103 casos/100.000 habitantes*.

Según la última información de mortalidad disponible a diciembre 2004, se ha informado el fallecimiento de 5.043 personas.

\section{Morbilidad}

En Chile, la tasa de notificación anual de infección por VIH/SIDA muestra una tendencia al aumento a través de los años, desde 0,05 casos/ 100.000 habtes. en 1984 a 9,9 casos/100.000 habtes en el 2004, lo que representa un incremento aproximado de 200 veces. Sin embargo, se observa una disminución en la velocidad de crecimiento a partir del año 1990.

La tasa de notificación en los hombres sigue el patrón de la curva nacional, con un marcado incremento entre el año 1984 y el 2004 ( 0,1 y 16,7/cien mil habtes, respectivamente). En las mujeres, la tasa de notificación es menor. El inicio de la epidemia en las mujeres fue en el año 1985, con una tasa de notificación de 0,02/100.000 habtes., llegando al 2004 a 3,2 casos/100.000 habtes (Figuras 1 y 2 ).

$\mathrm{Al}$ analizar por separado la infección por VIH y el SIDA, la tasa nacional de notificación de la primera se ha incrementado bruscamente desde 0,1 casos $/ 100.000$ habtes. en 1985 a 4,5 casos/100.000 hab. el año 2000 . El 2002 se produjo un aumento (5,6 casos/100.000 habtes) y tiende a disminuir los últimos dos años.

Desde $1984-0,05$ casos/100.000 habtes -se observa un aumento pronunciado de SIDA en el país hasta el

*Tasa calculada con población a mitad de período de la epidemia. 
año 1991 -2,6 casos/100.000 habtes-, momento en que la velocidad de crecimiento se lenteció, para luego estabilizarse desde 1996 en adelante, con tasas en el orden de 3 casos/100.000 habtes (Figura 2).

En el año 1997 se implementó la biterapia en Chile, y como ha sido demostrado en experiencias internacionales ésta podría llegar a tener sólo un efecto transitorio (MINSAL, 2002). Nuestro país incluyó la triterapia a partir del año 2000, logrando una cobertura en el sector público de $100 \%$ el año 2003. La infección por VIH y el SIDA fueron incorporados al GES (Garantías Explícitas en Salud) en modalidad piloto el año 2004, e incluidos definitivamente al año siguiente. Este régimen garantiza el acceso a la atención pública y privada de los pacientes diagnosticados con infección por VIH en un tiempo determinado. En términos de oportunidad, el paciente que necesite terapia anti-retroviral (TARV) puede acceder a ésta en forma precoz. También se protege financieramente al paciente, determinando desde gratuidad hasta un co-pago máximo, de acuerdo a su situación socioeconómica (MINSAL, 2004).

Por lo anterior, es importante realizar un seguimiento de estos pacientes de manera de establecer los impactos que tendrá el GES, y fundamentalmente la TARV en los efectos clínicos-inmunológicos (recuentos de linfocitos T- LT CD4), virológicos (carga viral$\mathrm{CV}$ ), aparición de enfermedades oportunistas, hospitalizaciones y retraso en aparición de la etapa SIDA. Otros impactos incluyen la letalidad y mortalidad, lo que finalmente tendrá un efecto socioeconómico en términos de ahorro en el Sistema de Salud (MINSAL, 2003).

\section{Distribución por sexo}

Del total de casos notificados entre los años 1984 y 2004, 85\% corresponden a hombres (Tabla 2, Figuras 3 y 4$)$.

\section{Distribución por grupos de edad}

Los grupos de edad más afectados están entre 20 y 49 años, concentrando $86 \%$ de los casos. No se observan diferencias significativas entre ambos sexos en relación a edad al momento del diagnóstico.

Con el fin de conocer la evolución de los grupos de edad más afectados, se calcularon las tasas de infección por VIH/SIDA por períodos quinquenales (desde 1984 hasta el 2003). Los resultados muestran (Tabla 3), que la tasa más alta en todos los períodos fue para el grupo de 20 a 39 años de edad, seguido por el grupo entre 40 y 59 años de edad.

La mediana de edad al momento del diagnóstico de la infección por VIH se ha mantenido estable, alrededor de 31 años, en los 20 años analizados (1984: 30 años; 2004: 32 años) (Figura 5).
La mediana de edad del diagnóstico de SIDA, por su parte, fue de 36 años (1984: 36 años; 2004: 37 años).

\section{Distribución geográfica}

Las tasas acumuladas más altas para el período 1984-2004 según región corresponden a las regiones Metropolitana, I', V' y II (Figuras 6 y 7, Tabla 4).

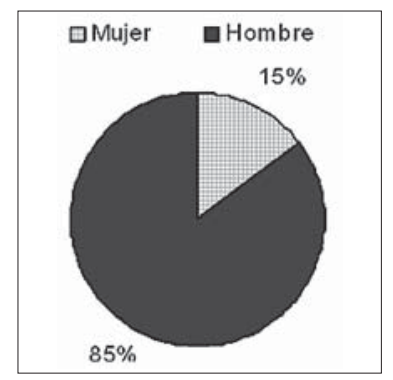

\begin{tabular}{|c|c|c|c|}
\hline \multirow[t]{2}{*}{ Clasificación } & \multicolumn{2}{|c|}{ Sexo } & \multirow[t]{2}{*}{ Total } \\
\hline & Mujer & Hombre & \\
\hline Infección por VIH & 1.370 & 6.188 & 7.552 \\
\hline SIDA & 777 & 6.063 & 6.840 \\
\hline Total & 2.147 & 12.251 & 14.398 \\
\hline
\end{tabular}

Figura 3. Distribución porcentual de casos de infección por VIH/ SIDA por sexo Chile, 1984-2004.

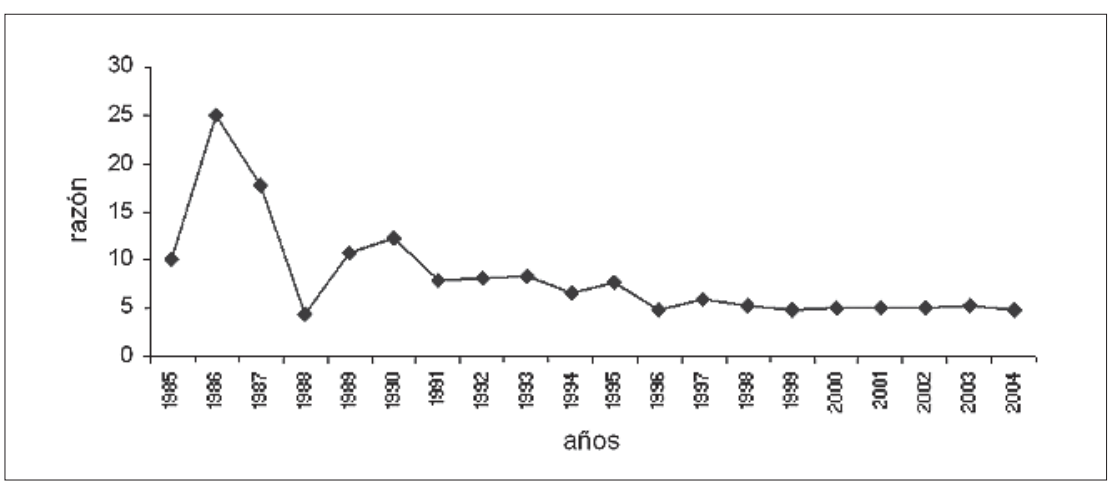

Figura 4. Razón H:M en VIH/SIDA. Chile, 1985-2004. La curva representa la razón H: M para cada año entre 1984 y 2004, donde se aprecia una estabilización de ésta desde 1996 en aproximadamente 5:1, es decir, por cada caso en mujer existen alrededor de 5 casos en hombres.

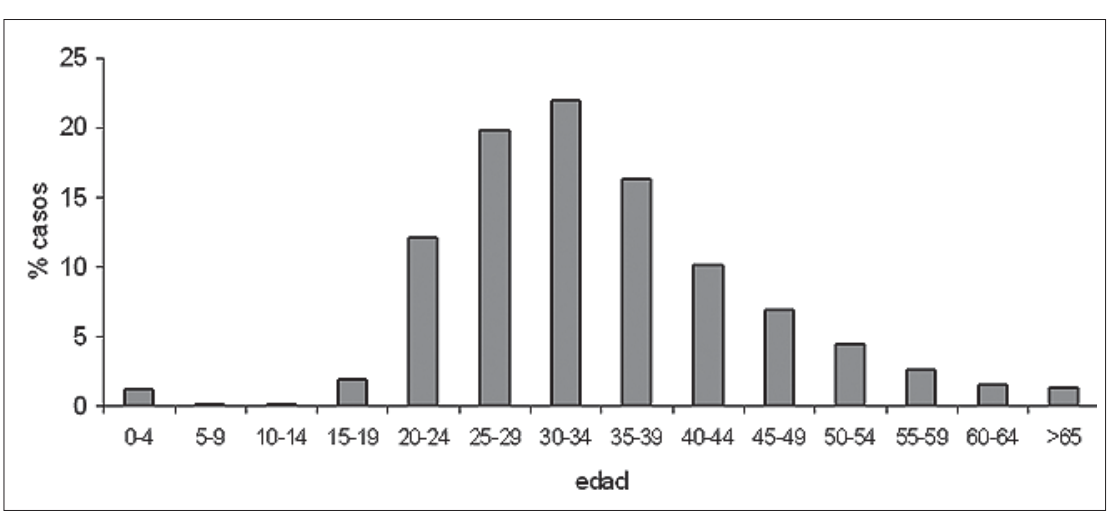

Figura 5. Distribución porcentual de casos de infección por VIH/SIDA según edad al diagnóstico Chile, 1984-2004. 
Tabla 3 . Casos y tasas de infección por VIH/SIDA según edad y años de diagnóstico. Chile, 1984-2004

\begin{tabular}{|c|c|c|c|c|c|c|c|c|}
\hline \multirow{2}{*}{$\begin{array}{l}\text { Año de } \\
\text { Diagnóstico }\end{array}$} & \multicolumn{2}{|c|}{ 0-19 años } & \multicolumn{2}{|c|}{ 20-39 años } & \multicolumn{2}{|c|}{ 40-59 años } & \multicolumn{2}{|c|}{$>60$ años } \\
\hline & Casos & Tasa & Casos & Tasa & Casos & Tasa & Casos & Tasa \\
\hline 1984-1988 & 12 & 0,24 & 170 & 4,18 & 59 & 2,82 & 5 & 0,48 \\
\hline 1989-1993 & 102 & 1,95 & 1613 & 35,30 & 467 & 19,42 & 65 & 5,34 \\
\hline 1994-1998 & 165 & 3,01 & 3055 & 62,65 & 903 & 31,78 & 95 & 6,76 \\
\hline $1999-2003$ & 278 & 4,98 & 4390 & 88,93 & 1605 & 46,91 & 185 & 11,36 \\
\hline 2004 & 67 & 1,20 & 899 & 24,50 & 403 & 10,60 & 49 & 2,70 \\
\hline
\end{tabular}

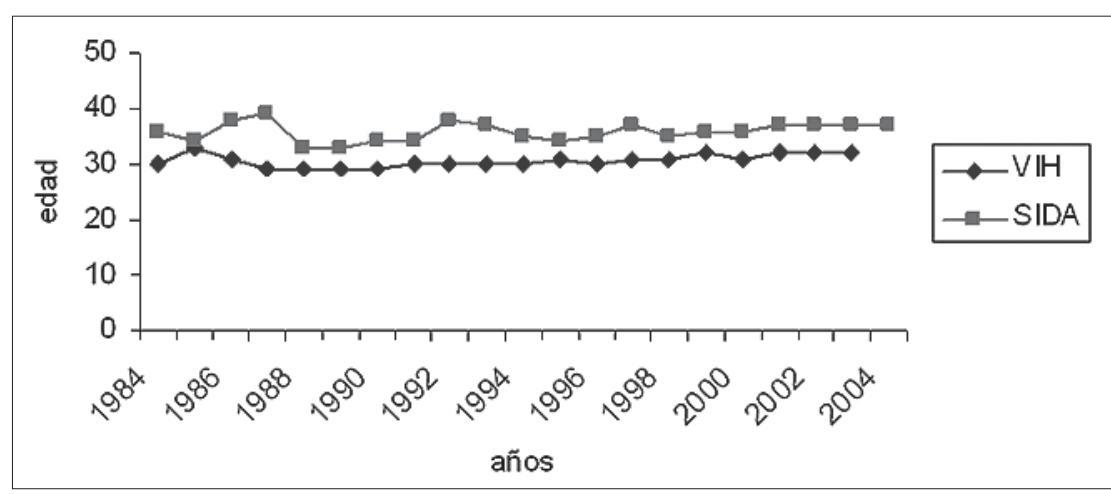

Figura 6. Mediana edad al diagnóstico de infección por VIH y SIDA. Chile, 1984-2004.

\section{Factores de riesgo}

La principal vía de exposición al virus fue la vía sexual, siendo la conducta homosexual y bisexual en 45 y 14\%, respectivamente) (Tabla 5, Figura 8).

La declaración de exposición heterosexual ha aumentado a partir del año 1996, mientras la homosexual se ha mantenido en niveles cercanos a $50 \%$. Paralelamente, han disminuido las otras vías no sexuales. Cabe destacar que $19 \%$ de los casos no declara su factor principal de exposición.

Las vías sanguíneas (transfusiones y aporte de factores de coagulación) se registran sólo en los primeros años de la epidemia, ya que se implementaron medidas de control en los bancos de sangre.

La transmisión vertical también ha presentado una fuerte disminución, principalmente por la incorporación en el año 1995 de las medidas preventivas en la transmisión madre a hijo, que incluyen el tratamiento a la madre durante el embarazo y, en el momento del parto, cesárea programada y lactancia artificial (Chávez et al., 2000).

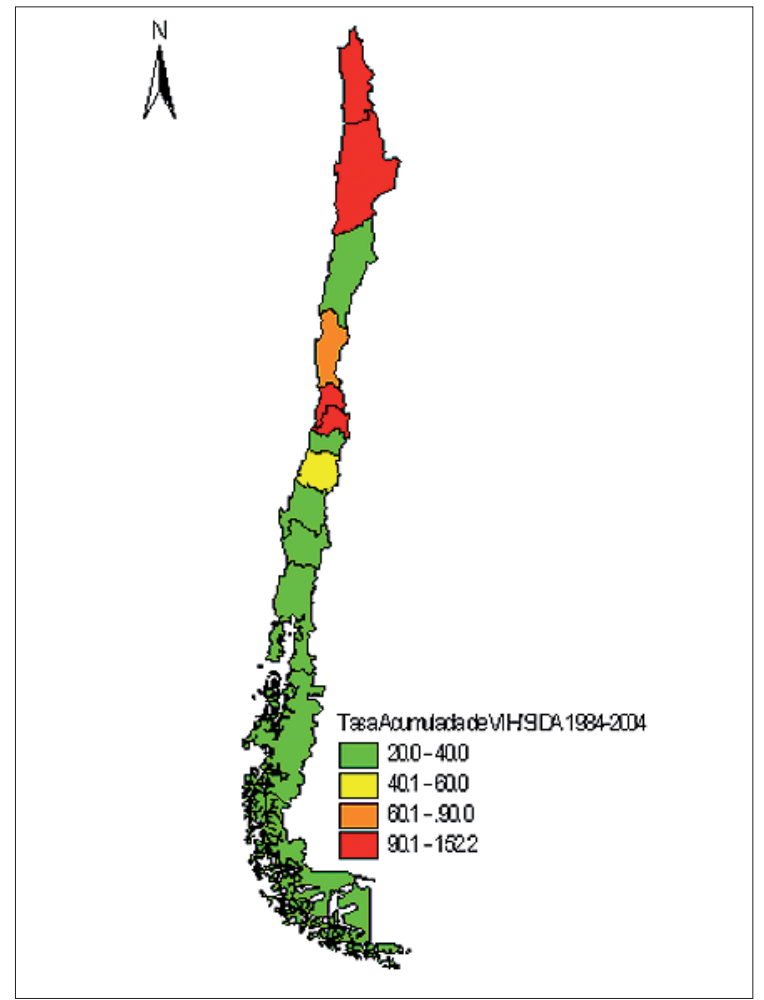

Figura 7.

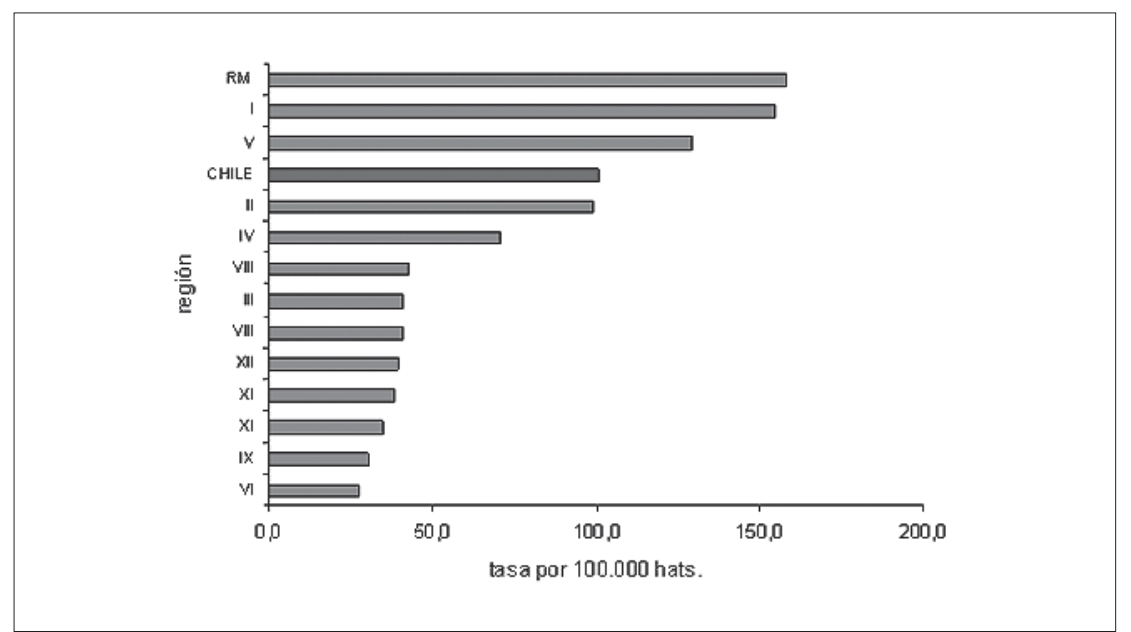

Figura 8. Tasa de casos de infección por VIH/SIDA según región. Chile, 1984-2004. 
Tabla 4. Vigilancia de infección por VIH/SIDA. Casos y tasa acumulada , según Región de notificación. Chile, 1984-2004. Región Casos y tasas de notificación

\begin{tabular}{|c|c|c|c|c|c|c|c|}
\hline & \multicolumn{3}{|c|}{ Casos } & \multicolumn{3}{|c|}{ Casos VIH/SIDA } \\
\hline & & VIH & SIDA & Total & Mujer & Hombre & Tasa \\
\hline I & Tarapacá & 310 & 266 & 576 & 90 & 487 & 154,5 \\
\hline 11 & Antofagasta & 257 & 188 & 445 & 80 & 366 & 99,1 \\
\hline III & Atacama & 64 & 36 & 100 & 29 & 72 & 41,4 \\
\hline IV & Coquimbo & 236 & 151 & 387 & 73 & 314 & 70,7 \\
\hline V & Valparaíso & 981 & 877 & 1.858 & 329 & 1.555 & 129,4 \\
\hline VI & Del Libertador & 127 & 78 & 205 & 33 & 172 & 27,7 \\
\hline VII & Maule & 193 & 184 & 377 & 94 & 293 & 42,9 \\
\hline VIII & Bío Bío & 340 & 407 & 747 & 114 & 634 & 41,2 \\
\hline IX & Araucanía & 130 & 125 & 255 & 60 & 195 & 30,8 \\
\hline$x$ & Los Lagos & 234 & 155 & 389 & 77 & 312 & 38,3 \\
\hline$X I$ & Aysén & 17 & 13 & 30 & 7 & 23 & 34,9 \\
\hline XII & Magallanes & 34 & 23 & 57 & 10 & 47 & 39,6 \\
\hline XIII & Metropolitana & 4.566 & 4.301 & 8.867 & 1.161 & 7.879 & 158,3 \\
\hline Total & I país & 7.489 & 6.804 & 14.293 & 2.157 & 12.349 & 101,0 \\
\hline
\end{tabular}

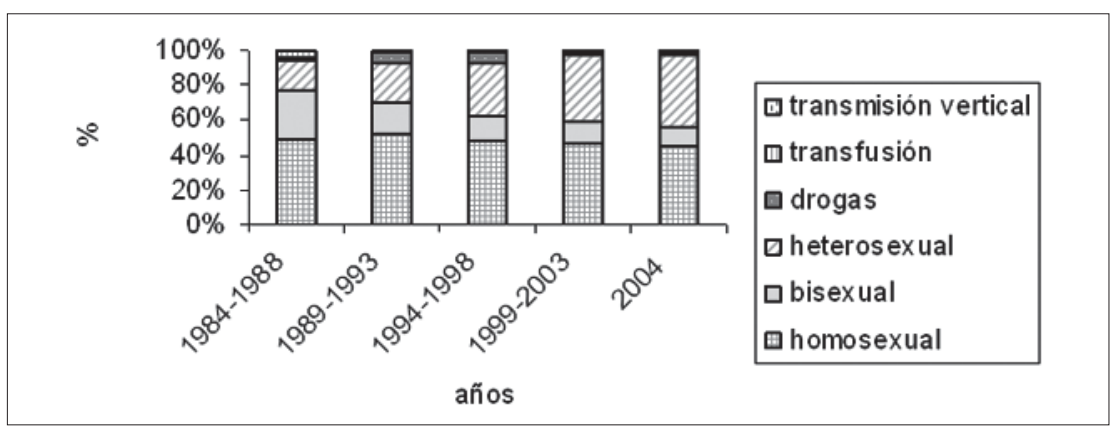

Figura 9. Distribución porcentual de casos de infección por VIH/SIDA, según factor principal de exposición. Chile, 1984-2004.

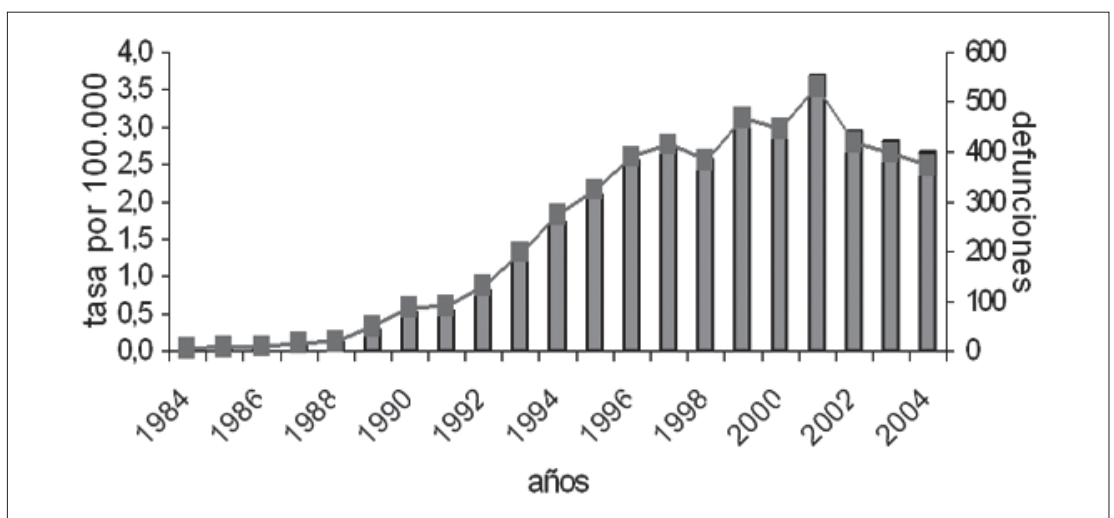

Figura 10. Número de defunciones y letalidad por SIDA. Chile, 1984-2004.

\begin{tabular}{|c|c|c|}
\hline Categoría & Casos & $\%$ \\
\hline Homosexual & 6.128 & 41,94 \\
\hline Bisexual & 1.841 & 12,60 \\
\hline Heterosexual & 4.229 & 28,94 \\
\hline Drogas & 387 & 2,65 \\
\hline Hemofilia & 29 & 0,20 \\
\hline Transfusión & 40 & 0,27 \\
\hline Transmisión vertical & 158 & 1,08 \\
\hline Desconocido & 1.799 & 12,31 \\
\hline Total & 14.611 & 100,00 \\
\hline
\end{tabular}

\section{Tabla 6. Casos de infección por VIH/SIDA según nivel de escolaridad. Chile, 1984-2004}

\begin{tabular}{|lrr|}
\hline Categoría & Casos & \multicolumn{1}{c|}{$\%$} \\
\hline Básica & 3.423 & 23,43 \\
\hline Media & 6.963 & 47,66 \\
\hline Ninguna & 299 & 2,05 \\
\hline Superior & 2.919 & 19,98 \\
\hline Desconocida & 1.007 & 6,89 \\
\hline Total & 14.611 & 100,00 \\
\hline
\end{tabular}

\section{Escolaridad}

El nivel de escolaridad alcanzado por los casos con infección por VIH/SIDA difiere del perfil observado en la población chilena, según el Censo 2002 (INE, 2002): la escolaridad media concentra un valor cercano a $50 \%$; la educación básica y superior es declarada por cerca de $20 \%$ de los casos respectivamente (Tabla 6). Esta distribución se ha mantenido estable.

\section{Mortalidad}

La proporción de defunciones por SIDA representó el año 2004, el 0,46\% del total de defunciones, proporción levemente inferior al año anterior $(0,51 \%)$.

Las primeras defunciones por SIDA en Chile ocurrieron en 1984, y desde entonces se acumulan hasta el año 2004, 5.043 muertes.

Tanto el número de defunciones como la tasa de mortalidad por SIDA se han incrementado, alcanzando su pico el año 2001, para luego descender el año 2004 en 30\% (Figura 9); esto podría explicarse por la incorporación de TARN de alta actividad (en inglés la sigla HAART).

La tasa de mortalidad en los hombres muestra el mismo perfil que la nacional, concentrando el $88 \%$ 


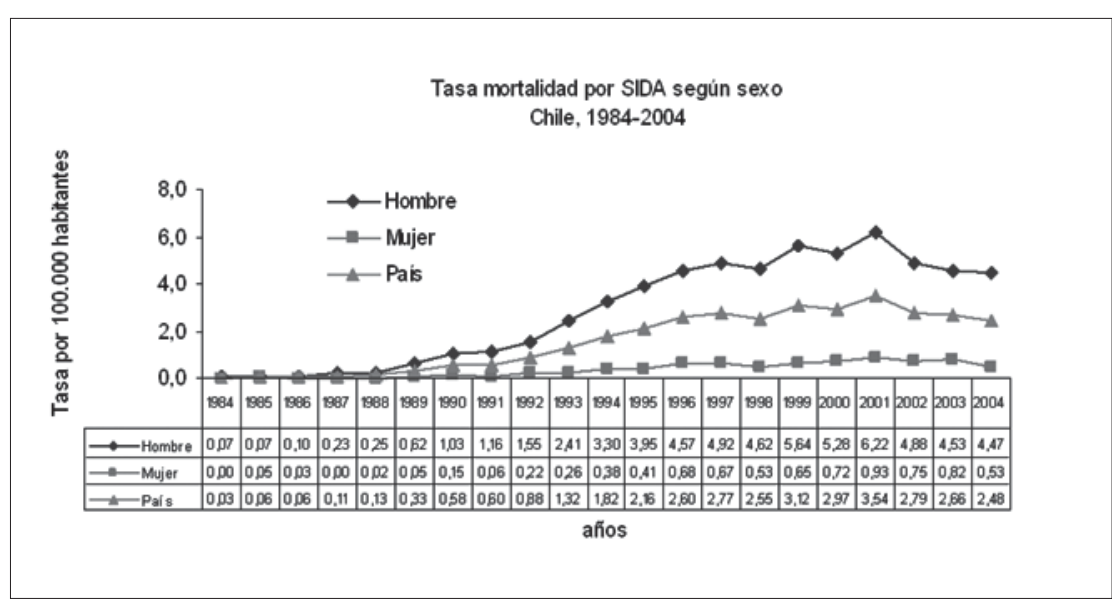

Figura 11. Tasas de mortalidad por SIDA según sexo. Chile, 1984-2004.

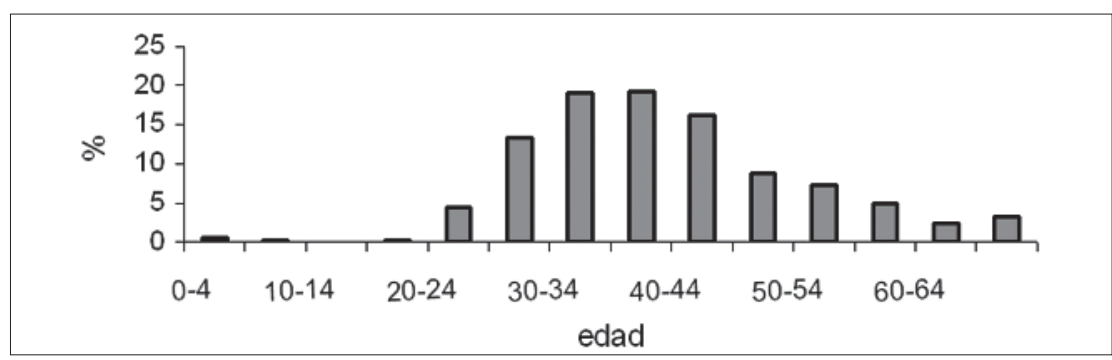

Figura 12. Distribución porcentual de defunciones por SIDA según edad. Chile, 1999-2004. progresión de la enfermedad, presentan niveles disminuidos de LT CD4 y finalmente fallecen (Wood, et al., 2003).

Entre los años 1999 y 2004, el grupo de 25 a 44 años concentró el $68 \%$ de las defunciones por SIDA, los grupos de 30a 34 y 35a 39 años concentraron alrededor de $20 \%$ cada uno, situación estable en el tiempo (Figura 11).

Con el objetivo de conocer la evolución de la edad de muerte por SIDA, se calcularon las tasas de mortalidad según edad al diagnóstico (por 100.000 habtes.), para los años 1997 al 2004. En cada uno de estos años, los grupos con las tasa más altas fueron los de 20 a 39 años y 40 a 59 años.

La letalidad del SIDA, tuvo niveles elevados los primeros años de la epidemia, disminuyendo de $66 \%$ en 1984 a 22\% el 2004 (Tabla 7).

En los primeros años de la epidemia, los casos de SIDA debutaron con enfermedades marcadoras graves y no se disponía de terapia efectiva. La situación anterior representó prácticamente la historia natural de la enfermedad (Figura 12).

Los años potencialmente perdidos (AVPP), es una estimación de la cantidad de años de vida que se pierden por muerte prematura (es decir, antes que la persona llegue a la edad en que se espera que muera). Se obtienen definiendo un límite potencial promedio de vida (80 años de edad, en este caso) y calculando la diferencia entre la edad a la defunción y ese límite.

El cálculo de AVPP por SIDA se incremento entre los años 1984 y 1999, alcanzando el mayor número en este último año (12.874 años de vida perdidos) (MINSAL, 2000). A partir del año 1999 se registró una disminución que se mantuvo hasta el año 2002, incrementándose el año 2003 en menor nivel que el año de mayor registro. Para el año 2004 los AVPP fueron 12.045 años, lo que implica una disminución respecto al año anterior de 3,8\% (total año 2003: 12.515 años de vida perdidos).

\section{Sistema de vigilancia epidemiológica}

El Decreto Supremo 158 (Ministerio de Salud, 2004), en su Art. 1, establece a la infección por VIH y el SIDA como enfermedad de notificación obligatoria diaria la que debe ser notificada por el establecimiento de salud a la Autoridad Sanitaria Regional, el mismo día que la persona reciba la confirmación diagnóstica de infección por VIH. La Secretaría Regional Ministerial(SEREMI) remitirá esta información al MINSAL una vez por semana, en la forma establecida por este último.

La vigilancia epidemiológica se define como el proceso continuo de recolección, análisis, e interpretación de datos, cuyo fin es detectar y actuar oportuna- 


\begin{tabular}{|c|c|c|c|c|c|c|c|c|}
\hline \multirow{2}{*}{$\begin{array}{l}\text { Año de } \\
\text { defunción }\end{array}$} & \multicolumn{2}{|c|}{ 0-19 años } & \multicolumn{2}{|c|}{ 20-39 años } & \multicolumn{2}{|c|}{ 40-59 años } & \multicolumn{2}{|c|}{ > 60 años } \\
\hline & Casos & Tasa & Casos & Tasa & Casos & Tasa & Casos & Tasa \\
\hline 1997 & 9 & 0,16 & 254 & 5,20 & 124 & 4,19 & 23 & 1,59 \\
\hline 1998 & 5 & 0,09 & 240 & 4,90 & 120 & 3,91 & 18 & 1,21 \\
\hline 1999 & 3 & 0,05 & 270 & 5,49 & 176 & 5,53 & 25 & 1,64 \\
\hline 2000 & 4 & 0,07 & 254 & 5,15 & 177 & 5,37 & 22 & 1,40 \\
\hline 2001 & 5 & 0,09 & 311 & 6,30 & 200 & 5,85 & 36 & 2,21 \\
\hline 2002 & 6 & 0,11 & 245 & 4,95 & 170 & 4,80 & 19 & 1,13 \\
\hline 2003 & 4 & 0,07 & 237 & 4,78 & 153 & 4,17 & 29 & 1,66 \\
\hline 2004 & 2 & 0,04 & 315 & 6,35 & 82 & 2,16 & 0 & 0,00 \\
\hline
\end{tabular}

mente sobre los problemas que suponen un riesgo para la salud de la población.

Existen diferentes modalidades de vigilancia, entre ellas, la Vigilancia Básica o Universal, que es la notificación obligatoria de todos los casos nuevos de las enfermedades que se vigilan bajo esta modalidad. Este es el sistema utilizado en Chile en el caso de infección por VIH/SIDA con una periodicidad de notificación diaria.

Forman parte del sistema de vigilancia de infección por VIH/SIDA los establecimientos de salud públicos y privados (ambulatorios u hospitalarios), laboratorios, Autoridad Sanitaria Regional (SEREMI), Ministerio de Salud (Departamentos de Epidemiología, Estadísticas e Información en Salud (DEIS) y la Comisión Nacional del SIDA (CONASIDA), e Instituto de Salud Pública (ISP).

El flujo de la información se inicia una vez que el médico tratante o la enfermera del Programa VIH/SIDA han recibido la confirmación del laboratorio de infección por VIH, comunicado al paciente el resultado y realizado el diagnóstico clínico por parte del médico. Se ingresa la información del paciente en el formulario ENO y en el formulario específico establecido por el MINSAL y se envían a la SEREMI de Salud correspondiente.

A partir del 2005, el DEIS del MINSAL realiza la recepción, digitación y validación de las notificaciones enviadas por las SEREMIS de Salud. La base de datos es analizada por el Departamento de Epidemiología y difundida a través de boletines trimestrales, que están disponibles en la página web de dicho departamento (http://epi.minsal.cl)

\section{Propuesta de actualización del sistema de vigilancia epidemiológica de infección por} VIH

La epidemia de infección por VIH/SIDA se ha modi-

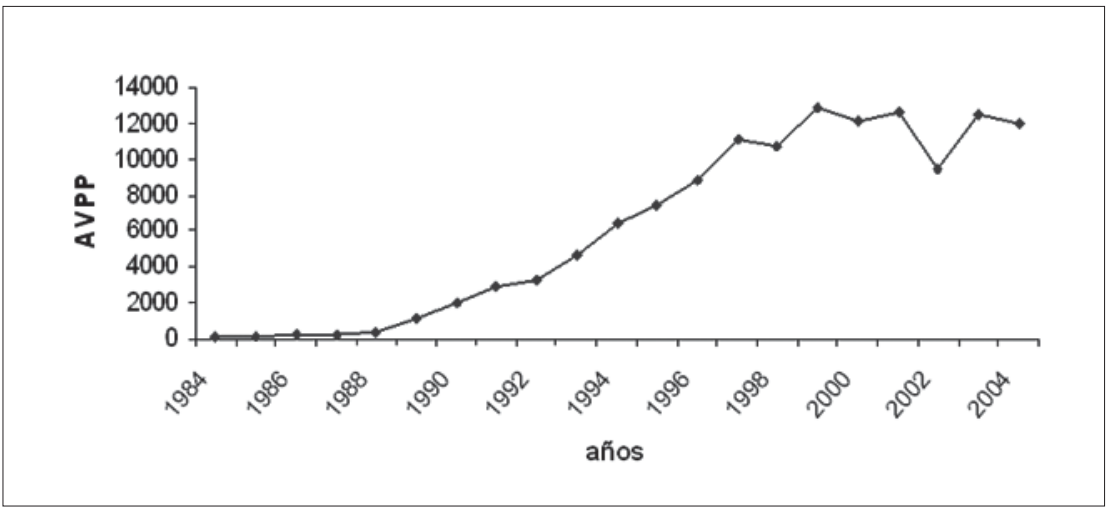

Figura 13. Años de vida potencialmente pérdidos (AVPP) por SIDA en hombres 20-49 años. Chile, 1984-2004.

ficado en el tiempo, requiriendo una revisión del sistema de vigilancia actualmente vigente en nuestro país, incorporando tanto la experiencia nacional como la internacional.

La propuesta planteada es un sistema integrado que contribuya a entender la dinámica de la epidemia de infección por VIH en el país, y proporcione información que permita diseñar planes de intervención y evaluación del impacto (ONUSIDA/OMS, 2002). Para ello, es necesario mejorar la calidad de la información.

La notificación se mantendrá a través de formularios ad hoc, que contemplan tanto la etapa diagnóstica de infección por VIH, como la de SIDA, además del Boletín ENO. Se espera avanzar en el sistema de notificación, mediante la creación de un formato electrónico de acceso restringido, en línea, para el envío de la información desde los niveles locales.

Una de las primeras actividades dentro del proceso de revisión de la vigilancia fue consensuar una definición de caso. Para ello, en abril del 2005, el Departa- 
mento de Epidemiología en conjunto con un grupo de expertos**, acordó la siguiente definición de caso de infección por VIH:

Caso confirmado: Toda persona cuyos resultados de las pruebas de laboratorio han sido confirmados por el Instituto de Salud Pública (MINSAL, 2001).

En términos generales, se acepta que es muy difícil establecer valores exactos de la epidemia de infección por VIH/SIDA, por lo que se sugiere comparar, complementar y triangular con otros datos adicionales, y de esta manera enriquecer el análisis de la situación epidemiológica. Se intensificará la utilización de registros tales como: mortalidad, morbilidad, datos de laboratorios, bancos de sangre, pruebas diagnósticas en mujeres embarazadas, egresos hospitalarios, registros de pacientes con TARV, encuestas de salud, encuestas de comportamiento, entre otros.

\section{Conclusiones y discusión}

El SIDA se describió en 1981 y a la fecha, la epidemia supera los 40 millones de personas infectadas, de las cuales 2,8 millones corresponden a personas bajo 15 años de edad. La distribución de los casos de infección por VIH/SIDA en el mundo muestra marcadas diferencias regionales.

En Chile, el primer caso de SIDA fue notificado en 1984 y desde entonces la notificación de casos ha ido en constante aumento. La mayor vía de transmisión del VIH registrada en nuestro país es la sexual. La epidemia nacional se caracteriza por afectar mayoritariamente a hombres en edades de plena actividad laboral y sexual.

Las políticas nacionales de acceso universal a los tratamientos anti-retrovirales ha permitido cambiar el rumbo de la historia natural de la enfermedad. Se ha logrado disminuir la letalidad causada por SIDA en $67 \%$ entre los años 1984 y 2004, y se espera que la mortalidad comience a descender.

La promoción activa de la vigilancia epidemiológica y el permanente monitoreo de la evolución de la epidemia constituyen, junto a la atención integral de las personas afectadas, pilares esenciales de las políticas públicas que buscan dar respuesta a la infección por VIH/SIDA. En este sentido, es fundamental determinar la prevalencia en la población general y en subpoblaciones especificas, como la tendencia de los principales factores de riesgo en relación con la población, el lugar y el tiempo que acompañan la transmisión del VIH.

\section{Resumen}

Desde 1984 a diciembre de 2004 se han registrado en Chile, según el sistema de vigilancia nacional, 14.611 casos de infección por VIH/SIDA ( $85 \%$ hombres) con una incidencia de 103/100.000 personas. La epidemia afecta mayoritariamente a hombres en plena actividad laboral y sexual. El principal factor de exposición ha sido la vía sexual. El acceso universal a tratamiento anti-retroviral (TARV) ha permitido cambiar el curso a la epidemia, en 1997 se implementó la bi-terapia y en 2003 la tri-terapia anti retroviral altamente activa. La letalidad global alcanzó a 2,4 casos/100.000 hbtes en el año 2004, lo que representa una reducción de $67 \%$ entre los años 1984 y 2004. La transmisión vertical ha disminuido también en forma significativa, gracias a la incorporación en el año 1995 de medidas preventivas que involucran tanto a la madre como al neonato. Actualmente se trabaja en la elaboración de un registro casos por vía electrónica como una manera de optimizar los recursos y contar con información en tiempo real de las estadísticas nacionales.

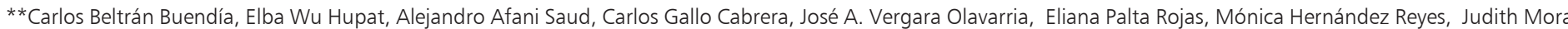

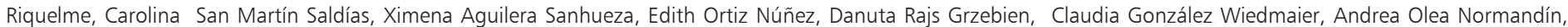
Eugenia Guerra Marchant, Roxana Barcena Vergara, Paulina Martínez Gallegos, Felipe Zuñiga Herranz, Franco Soto Cárdenas.
} 


\section{Referencias}

1.- Centers for Disease Control and Prevention (CDC). Trends in HIV and AIDS epidemic. 1998.

2.- Chávez A, Álvarez A, WU E. Transmisión vertical de la infección por virus de inmunodeficiencia humana: impacto de la aplicación del protocolo ACTG 076 en Chile. Rev Chil Infectol 2000; 17 (4) [en línea] http://www.scielo.cl/scielo.php?pid= S0716-10182000000400003 \&script $=$ sci arttext\&tlng=es (accedido: 2 mayo de 2006).

3.- Díaz H, Ruibal I, y Sánchez J. Fracaso del tratamiento antirretroviral y terapias de salvamento. Revisión actualizada. Rev Cubana Med 2003; 42 (4) [en línea] http:// www.bvs.sld.cu/revistas/med/vol42_4_03/ med07403.htm (accedido: 3 mayo de 2006).

4.- Instituto Nacional de Estadísticas (INE). Censo 2002.

5.- Joint United Programme on VIH/SIDA (UNAIDS) /World Health Organization (WHO).Guidelines for effective use of data from HIV surveillance systems. 2004.
6.- Ministerio de Salud (MINSAL). Boletín Epidemiológico $\mathrm{N}^{\circ}$ 13. 2000. [en línea] http://www.conasida.cl/bol/bol13/bol13.htm (accedido: 5 abril de 2006).

7.- Ministerio de Salud (MINSAL). Resolución Exenta $\mathrm{N}^{\circ}$ 371, febrero 2001.

8.- Ministerio de Salud (MINSAL)/ Comisión Nacional del SIDA (CONASIDA). Boletín epidemiológico $\mathrm{N}^{\circ}$ 14. 2002.

9.- Ministerio de Salud (MINSAL)/ Comisión Nacional del SIDA (CONASIDA). Boletín epidemiológico $\mathrm{N}^{\mathrm{o}}$ 15. 2003.

10.- Ministerio de Salud (MINSAL). Ley 19.966 de régimen general de garantías en salud. Chile 3 de septiembre 2004. [en línea] http: www.minsal.cl (accedido: 5 abril de 2006).

11.- Ministerio de Salud (MINSAL). Reglamento sobre notificación de enfermedades transmisibles de declaración obligatoria. 2004.

12.- Ministerio de Salud (MINSAL). Guías clínicas GES 2005. [en línea] http: www.minsal.cl (accedido: 5 abril de 2006).

13.- ONUSIDA/OMS. Grupo de trabajo de ONUSIDA/OMS para la Vigilancia Mundial del VIH/SIDA e ITS. Guías sobre la vigilancia del VIH de segunda generación. 2002
14.- Organización Internacional del trabajo. (OIT). El VIH/SIDA y el mundo del trabajo: estimaciones a nivel mundial, impacto y medidas adoptadas-2004 [en línea] http:// www.oit.org/public/english/protection/trav/ aids/publ/global_est_es/index.htm (accedido 5 abril de 2006).

15.- UNAIDS/WHO/FHI. UNAIDS/WHO

Working group on global HIV/AIDS and STI surveillance. The pre-surveillance assessment. Guidelines for planning serosurveillance of HIV, prevalence of sexually transmitted infections and behavioural components of second generation surveillance of HIV. 2005

16.- Wood E, Hogg R, Yip B, Harrigan R, O'Shaughnessy M., Montaner J. Is there a baseline CD4 cell count that precludes a survival response to modern antiretroviral therapy? AIDS 2003; 17 (5): 711-20, March 28 [en línea] http://www.aidsonline. com/pt/re/aids/abstract.00002030-2003032 8000009.htm; jsessionid $=$ GkCSYKvCNKR 4RvrnBwB50hygQmnD0znk3m4CKbGc5XK ZYVCBYDLc!1734750035!949856144!8091!-1 (accedido 12 mayo de 2006). 